\title{
Neuronal Nitric Oxide Synthase (NOSI) Polymorphisms Interact with Financial Hardship to Affect Depression Risk
}

\author{
Jane E Sarginson*,', JF William Deakin', lan M Anderson', Darragh Downey', Emma Thomas', \\ Rebecca Elliott' ${ }^{\prime}$ and Gabriella Juhasz ${ }^{1,2,3}$ \\ 'Neuroscience and Psychiatry Unit, Faculty of Medical and Human Sciences, University of Manchester, Manchester, UK; ${ }^{2}$ Department of \\ Pharmacodynamics, Faculty of Pharmacy, Semmelweis University, Budapest, Hungary; ${ }^{3}$ MTA-SE Neuropsychopharmacology and Neurochemistry \\ Research Group, Hungarian Academy of Sciences, Semmelweis University, Budapest, Hungary
}

\begin{abstract}
There is increasing evidence that genetic factors have a role in differential susceptibility to depression in response to severe or chronic adversity. Studies in animals suggest that nitric oxide (NO) signalling has a key role in depression-like behavioural responses to stress. This study investigated whether genetic variation in the brain-expressed nitric oxide synthase gene NOS I modifies the relationship between psychosocial stress and current depression score. We recruited a population sample of 1222 individuals who provided DNA and questionnaire data on symptoms and stress. Scores on the List of Life-Threatening Experiences (LTE) questionnaire for the last year and self-rated current financial hardship were used as measures of recent/ongoing psychosocial stress. Twenty SNPs were genotyped. Significant associations between eight NOS I SNPs, comprising two regional haplotypes, and current depression score were identified that survived correction for multiple testing when current financial hardship was used as the interaction term. A smaller three-SNP haplotypes (rs 10507279, rs 1004356 and rs37822 I 8) located in a regulatory region of NOSI showed one of the strongest effects, with the A-C-T haplotype associating with higher depression scores at low adversity levels but lower depression scores at higher adversity levels $(p=2.3 \mathrm{E}-05)$. These results suggest that NOSI SNPs interact with exposure to economic and psychosocial stressors to alter individual's susceptibility to depression.
\end{abstract}

Neuropsychopharmacology (20I4) 39, 2857-2866; doi:I0.1038/npp.20 I4. I37; published online I6 July 20 I4

\section{INTRODUCTION}

Depression is one of the most common forms of mental illness and even mild forms can impact adversely on quality of life, in addition to extracting a wider financial toll in terms of lost man-hours and treatment expenditure. A 2007 survey of adult psychiatric morbidity (McManus et al, 2009) estimated that $17.6 \%$ of adults in England have some forms of common mental disorder (CMD), with over half ( $9 \%$ of total) of the respondents with CMDs presenting with mixed anxiety and depressive disorder, which at the time was estimated to account for $20 \%$ of lost working days (DasMunshi et al, 2008). This report also highlighted a link between financial hardship and depressive episodes, with depression rates rising from $0.2 \%$ in men in the highest adjusted income quartile to $6.9 \%$ in the lowest. A number of studies that have suggested that recent or current financial hardship has a role in the initiation or maintenance of depressive episodes, over and above the effects of other

\footnotetext{
*Correspondence: Dr JE Sarginson, Neuroscience and Psychiatry Unit, Faculty of Medical and Human Sciences, Manchester University, Stopford building, Oxford road, Manchester MI3 9PT, UK, Tel: +44 (0) I6I 275 1728, E-mail: Jane.sarginson@manchester.ac.uk Received 22 February 20।4; revised II May 20I4; accepted 12 May 2014; accepted article preview online II June 2014
}

measures of socioeconomic position and demographic characteristics, which are known to be associated with the development of depression and related disorders (Butterworth et al, 2009, Meltzer et al, 2010, Hosang et al, 2012). In addition, unlike many other psychosocial stressors the effect appears to be consistent across both sexes (Maciejewski et al, 2001). This may be because financial hardship is acting as a proxy for other forms of deprivation and social stress, linking it more closely to personal survival than other measures of stress. There is also evidence that early socioeconomic stress is associated with epigenetic changes in the serotonin transporter (Beach et al, 2014), supporting the idea that genetics, epigenetics, and past environmental exposures interact to alter our susceptibility to environmental influences.

The nitric oxide (NO) pathway has a major role in neuronal signaling. In humans NO is produced by three nitric oxide synthases (NOS). The most abundant form in the brain is neuronal NOS (NOS1), which has a broad role in synaptic signaling as well as being implicated in learning, memory, neuronal plasticity and a number of psychiatric disorders, including depression (Steinert et al, 2010, Doucet et al, 2012), and schizophrenia (Weber et al, 2013). More specifically, NOS1 has a role in the regulation of the serotonin pathway (Pogun and Kuhar, 1994) and the HPA axis (Zhou et al, 2011) and is coupled to $\mathrm{Ca}^{2+}$-permeable $\mathrm{N}$-methyl-D-aspartate (NMDA)-receptors (NMDAR) at the 
postsynaptic density (PSD-95), through their mutual PDZbinding motifs (Weber et al, 2013). It is also stressresponsive, with chronic stress increasing NOS-I expression in many parts of the brain, including the hippocampus in animal models (Joca et al, 2007, Zhou et al, 2011). A number of animal experiments report that NOS-inhibiting drugs exert antidepressant-like behavioural effects in stress models (Silva et al, 2012).

SNPs in the NOS1 region (12q24) have been associated with major depression (McGuffin et al, 2005, Galecki et al, 2011) and with general psychological distress in a large multicentre GWA meta-analysis (Luciano et al, 2012). The present study tested the prediction that the influence of genetic variation in the NOS1 gene on current depression would be more apparent when tested in relation to recent/current adversity rather than in isolation. It also compared results obtained using a simple five-point selfreport current financial hardship scale as a measure of current psychosocial stress to those obtained using recent (within the last year) List of Life-Threatening Experiences (LTE) scores, as the LTE has been shown to have a high test-retest reliability and good correlation with more detailed semistructured life event interviews (Brugha and Cragg, 1990). The study cohort is based on a population sample recruited for the NewMood (New Molecules in mood disorders) study, which was designed to identify genes associated with depression and other mood disorders (Juhasz et al, 2009).

\section{MATERIALS AND METHODS}

\section{Study Cohort}

The study was approved by the local Ethics Committee and was carried out in accordance with the Declaration of Helsinki. All participants provided written informed consent. Subjects aged 18-60 years were recruited, predominantly from Greater Manchester, United Kingdom, as a population sample through general practices and a website (http://www.newmood.co.uk). Full details of the recruitment strategy, data collection, including how all the measures were derived and validated can be found in the Supplementary Information and has been published previously (Juhasz et al, 2009).

A questionnaire pack and genetic sampling kit were sent to the participants by post. The questionnaire contained a background questionnaire (Juhasz et al, 2009), which included demographic, health and lifestyle measures, and an inventory of the individual's personal psychiatric history. Depression within the last week was measured using the depression subscale of the self-report 53-item Brief Symptom Inventory (Derogatis, 1993).

Current financial hardship was assessed using a self-report five-point scale $(1=$ living very comfortably, $2=$ living quite comfortably, $3=$ just getting by, $4=$ finding it difficult to make ends meet, $5=$ not able to make ends meet). The List of LTE within the last year was used to measure recent negative life events (Brugha and Cragg, 1990). In the post hoc analysis four subsets of the LTE were considered, consisting of questions relating to intimate relationships (Category 1; Q5 and 6), finance and employment (Category 2; Q8-10), illness/injury/problems (Category 3; Q1, 7, 11, and 12), social network, including loss of partner (Category 4; Q2-4). Full details can be found in Brugha et al (1985) and Rijsdijk et al (2001).

Individuals who reported manic or hypomanic episodes, psychotic symptoms, or obsessive-compulsive disorder were excluded and the experimental cohort was limited to individuals of self-reported Caucasian ancestry as this was the largest ethnic group, producing a cohort of 1222 (Supplementary Table S1).

\section{SNP Selection and Genotyping}

Genomic DNA was extracted using the Freeman et al (2003) protocol from buccal mucosa cells collected by cytology brush (Cytobrush plus C0012; Durbin PLC). The SNPs (Table 1) were selected based on the International HapMap Project Phase I. June 2005 release (http://hapmap.ncbi.nlm.nih.gov/, CEU population) using the HaploView software (http://www.broad.mit.edu/personal/jcbarret/haploview/). The 20 selected SNPs were estimated to impute $\left(r^{2}>0.8\right)$ $37 \%$ of the SNPs in the gene region $(65-\mathrm{kb}$ downstream and $5-\mathrm{kb}$ upstream of gene to include potential regulatory regions) using an updated HapMap release (hapmap_rel27), with the majority of the coverage focused on the exons and regulatory regions. Genotyping was carried out using the IplexTM assay from Sequenom MassARRAY technology (Sequenom, San Diego) following the manufacturer's protocol (http://www.sequenom.com). Genotyping was carried out blind to phenotype. A $15 \%$ replication of genotyping was built into the study design, from which an overall type I error rate of $0.016 \%$ was calculated.

\section{Statistical Analysis}

Statistical analysis was carried out using $\mathrm{R}$ Commander (Fox, 2005). Owing to the sometimes modest number of minor homozygotes all primary analyses were carried out using both additive (Add) and dominant (Dom; minor homozygotes grouped with the heterozygotes) genetic models. A false discovery rate (FDR; Benjamini and Hochberg, 1995) of 5\% (Q-value: http://genomics. princeton.edu/storeylab/ qvalue/) was used to produce $p$-values corrected for multiple testing. As LTE and financial hardship were to be compared as measures of current psychosocial stress, the models containing them were corrected separately.

Significant skewing of the distribution of potentially confounding factors between genotypic groups was checked for in sex, age, marital status, employment status, education, housing situation, long-term health issues, current general health, financial hardship, LTE, social support problems, and childhood adversity.

The statistical analysis looked at the effect of genotype stress interaction on depressive symptom score. In all analysis current depression score was used as the outcome measure and the analysis was performed separately for LTE score (within the last year) and the five-point current financial hardship. As would be expected in a population sample, the BSI depression score has a non-normal distribution with $19 \%$ of participants reporting no depressive symptoms (Supplementary Information), a Poisson regression model was used with age and sex included as covariates. 
Table I SNP Location, HWE, and Frequency Information

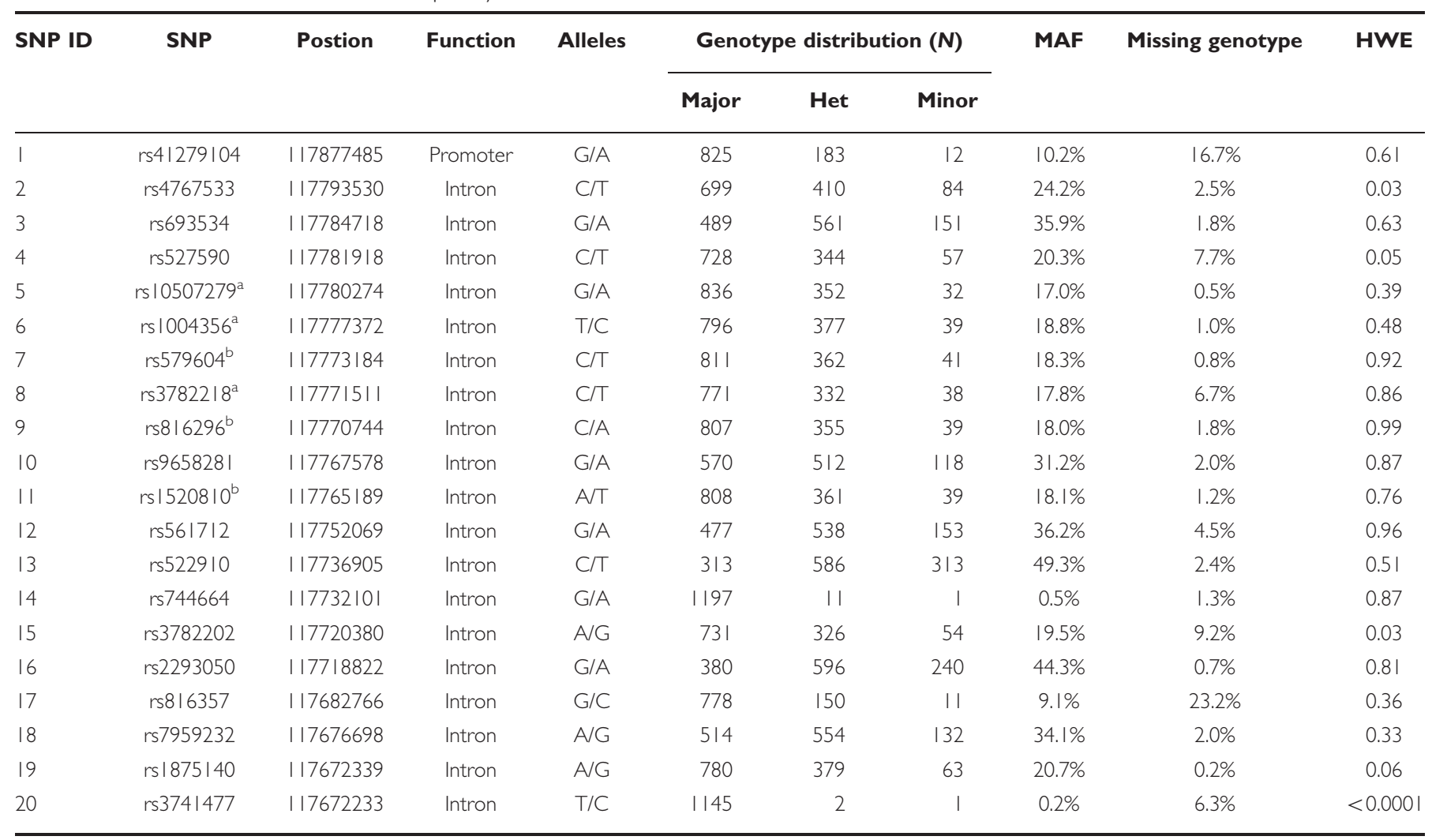

Abbreviations: HWE, Hardy-Weinberg equilibrium; MAF, minor allele frequency.

${ }^{a, b}$ Alleles (major/minor) a and $b$ indicate two separate sets of SNPs in strong linkage disequilibrium $\left(r^{2}>0.8\right)$. NOSI is located at I2q24.22, SNP position is taken from dbSNP build 138.

Haplotypes were created using JMP Genomic v5's (Cary, NC, USA) Haplotype Estimation tool. Individuals with a final expectation maximization probability of less than 0.8 were excluded and haplotypes with frequencies of less than $5 \%$ were grouped. Additional linkage disequilibrium (LD) analysis was carried out using Haploview v4.2 (Broad Institute, Cambridge, MA, USA). In addition to haplotypic trend regression gene $\times$ environment interaction analyses, as described above, were repeated for each haplotype separately.

The function/potential function and evolutionary conservation of regions showing associations to the outcome measure was examined using ENCODE (Encyclopedia of DNA Elements) project data via the UCSC genome browser (http://genome.ucsc.edu/ENCODE/) and the evolutionary conserved region (ECR) browser (http://ecrbrowser.dcode. org/).

Additional post hoc analysis was carried out to check for sex-based biases and to examine the impact of specific LTE question subsets, including comparing individual responses to the five-point financial hardship scale and the LTE question 'Have you suffered from a major financial crisis?'

\section{RESULTS}

\section{Demographic and Baseline Analysis}

Analysis of each SNP's properties resulted in the exclusion of the NOS1 SNPs rs3741477 and rs744664 from further analysis because of their low MAF within this study cohort $(<1 \%)$. In addition two SNPs, rs41279104 and rs816357, had over a $10 \%$ genotyping failure rate and were excluded from the haplotypic analysis. Three SNPs showed moderated but significant variation from Hardy-Weinberg equilibrium (Table 1).

No associations between genotype and demographic or lifestyle measures were identified, which passed an uncorrected significance threshold of $p=0.01$, except for an association between rs9658281 and LTE $(p=0.004)$. LD analysis (Figure 1) identified two sets of three SNPs in strong LD $\left(r^{2}>0.8\right.$; Haplotype A (HapA): rs10507279-rs1004356rs3782218; Haplotype B (HapB): rs579604-rs816296rs1520810). Supplementary Figure 1 shows regional D'.

\section{Single SNP Analysis}

Significant associations between eight NOS1 SNPs and current depression (Table 2), with financial hardship as an interaction term, were identified that survived correction for multiple testing, using both additive and dominant models (rs693534, rs10507279, rs1004356, rs3782218, rs9658281, rs561712, rs522910, and rs2293050). Three of these SNPs (rs693534, rs561712, and rs2293050) also showed weaker but significant associations with LTE score as the interaction term. No significant associations were identified when financial hardship or LTE score were not included as an interaction terms. 


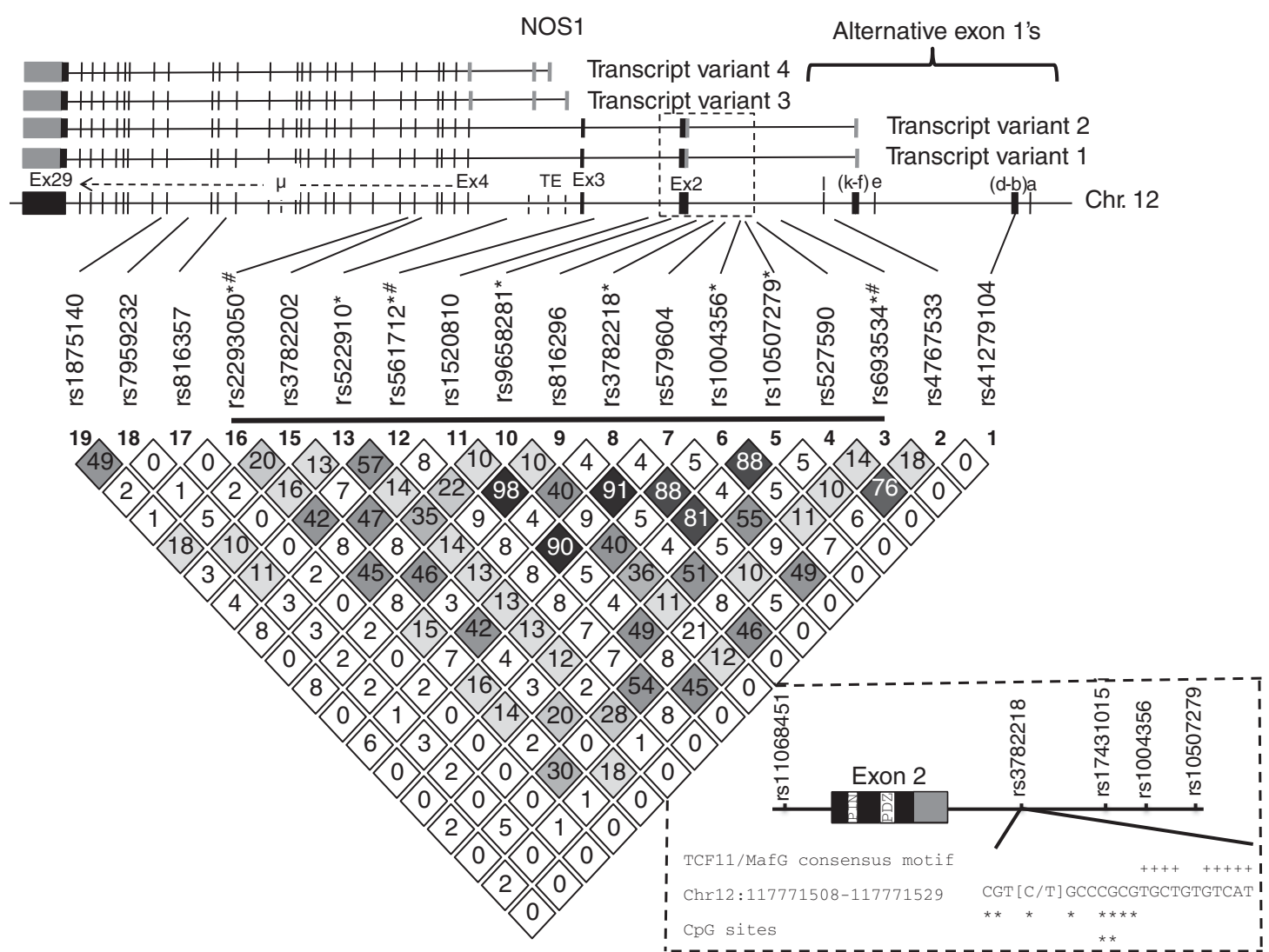

Figure I Schematic of the pairwise LD $\left(r^{2}\right)$ for this study cohort, with extremely low MAF SNPs $(<1 \%)$ excluded. The position of the SNPs is also shown in relationship to common NOSI transcripts. Both NOSI show complex tissue-specific regulation. NOS I has I2 alternative exon I's (a-l) with variant I

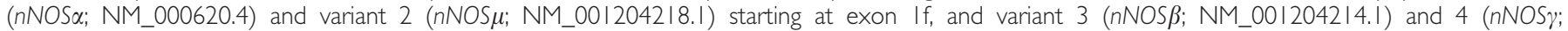
NM_00 I 2042 I3.I) initiating at alternative start points in intron 3 (Bros et al, 2006, Steinert et al, 20 I0). The inserted schematic shows in greater detail the HapA SNPs in relationship to selected regulatory and function elements in exon 2 and part of intron I. The inserted schematic is not to scale. rs56I7I 2 is located close to exon 3 and near proposed testis-specific exons (TE; Wang et al, 1997). Supplementary Figure I shows D'. Main key: Black, coding region; grey, untranslated regions (UTR); - an alternative tissue-specific exon; solid black line indicates those SNPs included in the regional haplotype; *significant with financial hardship as the interaction term; ${ }^{*}$ significant with LTE as the interaction term. Insert key: PIN, site of action for the protein inhibitor of NOS I (PIN); PDZ, the PDZ-binding motif allows NOSI to bind to different proteins and the plasma membrane; i, indicates the imputed SNPs imputed by HapA SNPs in the HapMap CEU cohort; +, the consensus sequence for the TCFI I/MafG transcription motif; *,potential CpG methylation sites; **, proven methylation sites (UCSF Brain DNA Methylation; www.genome.ucsc.edu).

Post hoc analyses using subsections of the LTE score showed that question 10 'Have you suffered from a major financial crisis?' produced the same pattern of significance as the 1-5 self-rated financial hardship scale. Analysis of the four LTE subcategories (Brugha et al, 1985, Rijsdijk et al, 2001) identified moderate associations for category 3 (Table 2), which covers serious personal illness/injury and legal or personal problems. No associations with intimate relationships (Category 1), finances/employment (Category 2), and social network, including loss of partner (Category 4), were identified (Results not shown).

\section{Haplotype Analysis}

To test the independence of the single SNP associations, haplotypes were created for the 66-kb region (13 SNPs; rs693534-rs2293050) containing the significant single SNPs from the primary current depression analysis. This produced five regional haplotypes with frequencies of greater than 5\% (Figure 2), with the minor alleles of the significant SNPs associating with the two most common haplotypes
(Hap1 and Hap2), demonstrating that the single SNP findings are not independent of each other. Standard haplotypic regression analysis with age and sex as covariates and financial hardship or recent LTE score, as covariates rather than interaction terms produced no positive associations. However, when the full model was run for each haplotype individually using an additive model, Hap $1\left(p^{\mathrm{FDR}}=1.3 \mathrm{E}-04\right.$, risk) and Hap2 ( $p^{\mathrm{FDR}}=1.8 \mathrm{E}-03$, protective) showed positive associations using financial hardship as the interaction term and Hap $1 \quad\left(p^{\mathrm{FDR}}=1.0 \mathrm{E}-04\right.$, risk $)$ for recent LTE score (Supplementary Table S2).

A comparison of the rates of increase in depressive symptoms with increasing financial hardship for all haplotypes with an estimated frequency of over $5 \%$ showed that carriers of two copies of Hap1 had the steepest increase in depressive symptoms and carriers of one copy of Hap2 $\left(p^{\mathrm{FDR}}=1.8 \mathrm{E}-03\right)$ and one copy of Hap1 showed the least increase (Supplementary Figure S2). Carriers of two copies of Hap2 showed a further decrease but only accounted for $1.8 \%$ of the cohort. Hap1 and Hap 2 are complementary, with the exception of five SNPs (rs527590, rs3782202, and 
Table 2 Single SNP Analysis of Current Depression Score

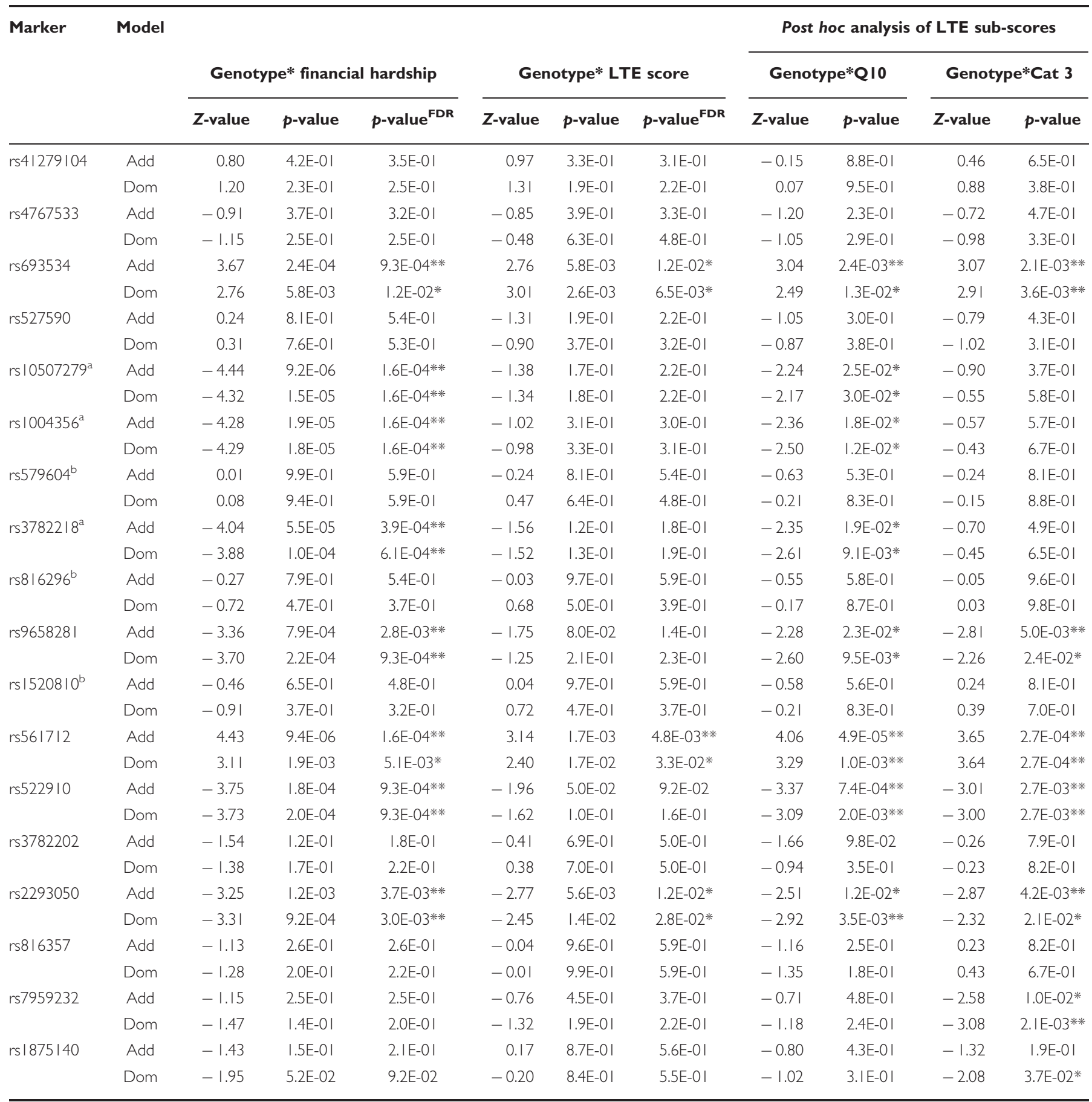

Abbreviations: Add, Additive model; Dom, Dominant model (minor homozygotes grouped with heterozygotes).

List of Life-Threatening Events (LTE) score question 10 reads 'Have you suffered from a major financial crisis?' LTE Category 3 covers illness, injury, and problems. Correction for multiple testing was performed using a 5\% false discovery rate (FDR), post hoc comparisons are uncorrected. Increasing financial hardship uses a I-5 rating scale. Current depression is measured using the brief symptom inventory (BSI) depression subscale

$a$ and $b$ indicate two separate sets of SNPs in strong linkage disequilibrium $\left(r^{2}>0.8\right)$.

*p-value FDR between 0.05 and 0.005 .

*** $p$-value FDR $<0.005$.

SNPs rs579604, rs816296, and rs1520810), which only discriminates for regional Hap4 (Figure 2).

These findings correlate well with the previous analyses as the minor rs 561712 allele is predicted to tag for $78.8 \%$ of
Hap1 and the minor alleles of rs10507279, rs1004356, and rs3782218 are similarly linked to Hap2 and these SNPs showed the strongest associations in the single SNP analysis (Table 2). As rs10507279, rs1004356, and rs3782218 are in 


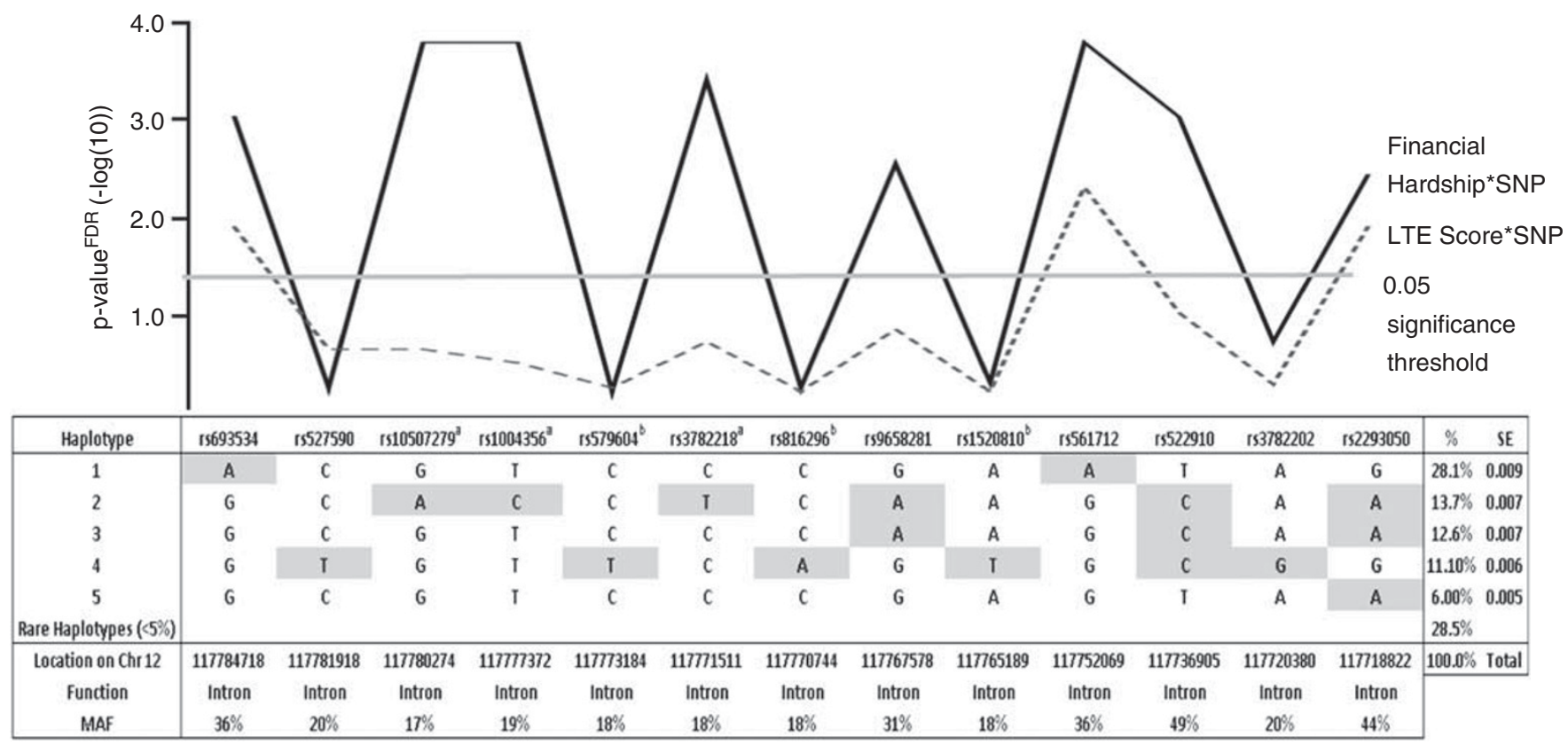

Figure 2 NOSI haplotypes and their relationship to single SNP findings. The haplotypes were created for the 13 SNPs located in the 66-kb region of NOSI, which contains all significant single SNP associations to current depression. Single SNP p-values corrected for multiple testing using a false discovery rate (FDR) of 5\% are shown as - log 10 . Results for both primary models are shown. The trait variable, mean current depression score, was calculated using the brief symptoms inventory (BSI), and age and sex were included as covariates. Financial hardship or recent LTE score were included as an interaction term and main effect. MAF, minor allele frequency. SNP ID, rs numbers can be found in Table I.

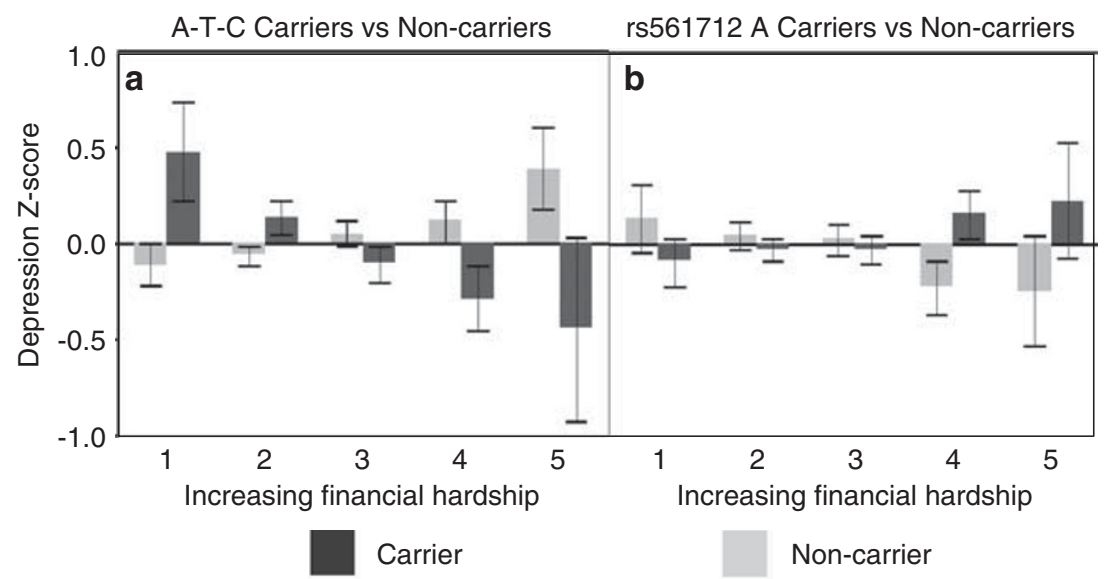

Figure 3 Effect of A-C-T and rs561712 minor A allele carrier status on variance from the mean for current depression score with increasing financial hardship. Both rs561712 A allele carriers and A-C-T carriers show an inversion of effect at financial hardship score 3, with the A-C-T carriers going having above mean to below mean and rs561712 A allele carriers showing the inverse. SE bars are included. The numbers in each subgroups according to financial

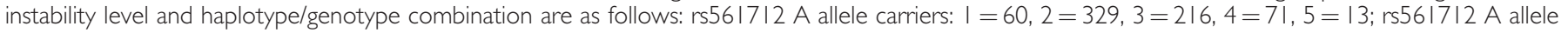
non-carriers: $I=41,2=29 \mid, 3=150,4=53,5=11$; A-C-T carrier: $\mid=24,2=154,3=102,4=32,5=5$; A-C-T non-carrier: $\mid=68,2=356,3=242$, $4=84,5=18$.

near complete LD with each other, it is appropriate to consider them together and they will now be referred to as HapA (G-T-C $=80.4 \%, A-C-T=16.2 \%$, rare haplotypes $=$ $3.4 \%)$. In $84.5 \%$ of cases, the minor A-C-T haplotype is associated with Hap2 and HapA showed a haplotypic $(p=2.3 \mathrm{E}-05$, Dom and Add) association with current depression when financial hardship was considered, which was equivalent to the associations seen for the single SNPs (Table 2). This association reflects the fact that A-C-T non-carriers had the smallest mean depression score at the lowest financial hardship score but showed a steeper increase in the mean depression score as financial hardship increased than A-C-T carriers (Figure 3a). This resulted in an apparent inversion of effect at financial hardship score 3, which corresponds to the answer 'just getting by'. This finding was mirrored by rs561712, with carriers of the minor A allele showing a more rapid increase in depression symptoms with increasing financial hardship (Figure $3 \mathrm{~b}$ ), 
this could be explained by HapA and rs561712, respectively, tagging for Hap2 and Hap1, which are complimentary.

\section{Sex Bias}

In all analyses sex, which was included in the model as a fixed variable, was significant. No potentially confounding associations between sex and genotype were identified during baseline analysis. However, in this study the female proportion of the cohort had a significantly higher $(p<0.0001)$ mean current depression score than the male portion (mean \pm SE; female, $1.2 \pm 0.04 v s$ male, $0.8 \pm 0.06$ ). The current depression model with financial hardship as the interaction term was run for each sex separately (Supplementary Table S3) with females $(n=849)$ producing a similar pattern of significant association to the full cohort and males $(n=373)$ producing significant associations for the core of haplotype 2 SNPs (rs10507279, rs1004356, rs3782218, and rs9658281).

\section{DISCUSSION}

There is increasing evidence that genetic factors influence the long-term effects of early adversity (Buchmann et al, 2013, Heim and Binder, 2012) but less work has been undertaken on current stress in adults. This study builds on previous reports associating NOS1 SNPs with current depression and psychological distress (McGuffin et al, 2005; Luciano et al, 2012) by demonstrating that the associations are likely to be mediated by responsivity to stress. The findings are compatible with findings in rodents implicating NOS1 in behavioural, neural, and endocrine responses to stress (Zhou et al, 2011). In humans, post-mortem studies have shown altered NOS1 levels in the anterior cingulate cortex (Gao et al, 2013) and hippocampus (Oliveira et al 2008) of depressed patients compared with non-depressed individuals, and the NOS1 SNP rs6490121 has been associated with changes in prefrontal cortical function and grey matter density (Rose et al, 2012).

The strongest effects in this study were obtained for the simple five-point self-rated scale of current financial hardship, with eight SNPs and the two regional haplotypes they tag for producing associations, which survive correction for multiple testing. LTE question 10 'Have you suffered from a major financial crisis?' showed a similar interaction with NOS1 genotype to the financial hardship scale but was less significantly associated to current depression (Table 2). This may reflect an advantage of the current financial hardship rating scale in measuring current financial stress as only $48 \%$ of those reporting that they were currently 'finding it difficult to make ends meet' $(n=129)$, and $76 \%$ of those reporting they were 'not able to make ends meet' $(n=25)$, considered that they had suffered from a 'financial crisis' in the last year.

Psychological stress is difficult to measure and there may be a number of explanations of why the findings for financial hardship are consistently higher than those for LTE, including that the financial hardship score is acting as a proxy for the deprivations and social difficulties that accompany financial hardship. Analysis of recent LTE score by subcategory only produced associations for Category 3
(Table 2), offering support to the idea that the type and timing of the psychological stress are important, as Category 3 covers serious personal illness/injury and legal or personal problems, which often have persistent or ongoing negative effects.

HapA and rs561712, which, respectively, tag for the regional haplotypes Hap2 and Hap1 the regional haplotype (Figure 2), both show what appears to be an inversion of effect at the level of current financial hardship score 3 ('just getting by'). Carriers of the minor A-C-T haplotype showed the most depressive symptoms when considering themselves financially stable, and the lowest depressive symptoms when they had moderate to severe financial problems (Figure 3a), whereas carriers of the minor rs561217 A allele showed the inverse pattern (Figure $3 \mathrm{~b}$ ).

Another inversion of effect in relation to stress has previously been observed for the functional NOS1 promoter VNTR (ex1f-VNTR), which is located by exon 1f, in a study into impulsivity in Estonian adolescents. The authors suggest that in the presence of an adverse environment the impulsivity associated with the short form of the exlfVNTR switched from adaptive to maladaptive, thus reversing its potential selective advantage (Reif et al, 2011).

NOS1 expression is subject to complex regulation and produces a number of different isoforms, which differ in time course and according to tissue, and the type of stimulus. (Bros et al, 2006). This regulation includes regulatory sites in intron 1 and inhibitory elements within exon 2 (Jaffrey and Snyder, 1996) that are located firmly within the region of interest identified by this study (Figure 1 insert), suggesting that the observed inversion of effect could be because of a disruption in the regulation of the gene. Exon 2 also contains an $\mathrm{N}$-terminal PDZ domain, which allows NOS1 isoforms containing exon 2 to bind to the plasma membrane and a number of different proteins (Bros et al, 2007). This includes the NMDA receptor, where it acts as a second messenger for the NMDA pathway (Weber et al, 2013). The NMDA pathway has repeatedly been associated with major depression (Mathews et al, 2012) and schizophrenia (Weber et al, 2013). In addition, the NMDAR/PSD95/NOS1 complex has been a target for novel treatments of depression (Doucet et al, 2012).

Interestingly, the HapA SNP rs3782218, which is located $2-\mathrm{kb}$ downstream from the start of exon 2 , is located in an ECR, which suggests a regulatory function. It is also close to an ENCODE-confirmed lympho enhancer (Pu.1) motif and disrupts one of the $\mathrm{CpG}$ sites in a $\mathrm{CpG}$ cluster directly next to the consensus portion of a predicted binding motif for TCF11/MafG (Johnsen et al, 1996), a heterodimeric transcriptional repressor (Russo et al, 2004), further supporting a regulatory role for this SNP (Figure 1 insert). The same binding motif is present in the promoter region of NOS2 and has been shown to repress the induction of NOS2 expression in response to the cytokine TGF-beta as part of the inflammatory response (Berg et al, 2007).

In addition, selective methylation of $\mathrm{CpG}$ sites has a role in transcriptional regulation and the CGTCG motif, which is disrupted by rs3782218, is predicted to have a high probability of being involved in methylation-based regulation (Lu et al, 2010). Recent studies have reported genespecific and global changes in DNA methylation in response to psychological stress in humans (Bick et al, 2012, Ursini 
et al, 2011). These potential explanations could help to explain the inversion of effect seen for HapA, as a reduction or alteration in regulatory control may be detrimental under normal environmental conditions but beneficial under more stressful conditions. In addition, rs3782218 has previously been associated with early-onset Parkinson's disease (Hancock et al, 2008), which has stress as a risk factor and has been linked to excess NO (Aquilano et al, 2008).

The associations identified in this study are reasonably strong and are not only consistent with previous genetic and functional findings but also suggest a mechanism for them. Nevertheless, the study has several limitations. All ratings are based on self-completed questionnaires, and are therefore subjective and may be influenced by recollection bias. However, we have previously reported highly significant correlations between self-report and interviewassessed mood ratings in a subset of the participants using independent trained investigators in this cohort (Juhasz et al, 2009). As ethnic ancestry was not confirmed by either interview or targeted genotyping, undetected population stratification may be an issue but is likely to be limited as participants were recruited from a single suburban geographical location. In addition, there is always a degree of self-selection by participants based on motivation levels and interest in the subject matter in any form of population sampling; in this study this resulted in high levels of participation by individuals with a self-reported history of depression, many of whom were women; therefore, the study may not accurately represent the population it was recruited from. Additional information on these issues can be found in the Supplementary Materials and Methods.

Ideally, the results should be replicated in an independent sample; however, partial replication is afforded by the presence of the findings in separate analyses within each sex. Furthermore, the similarity of the interaction between genotype and both measures of recent adversity on current depression point to internal consistency. In addition, the low frequencies of some SNPs resulted in there being few carriers in the extreme adversity groups. Replication in a large independent cohort and functional analysis of rs3782218 would be advisable.

In conclusion, economic stressors are known to increase the risk of depressive episodes (McManus et al, 2009, Meltzer et al, 2010, Hosang et al, 2012) and this study may offer an insight into a potential biological mechanism for vulnerably to this type of stress. It also supports the 'differential susceptibility' hypothesis, which takes a more plastic approach to 'risk' alleles suggesting that under the right conditions these alleles may actually be beneficial (Belsky et al, 2009), such as in this case when the high-stress risk allele is associated with lower depression risk in the absence of financial hardship. Similar findings have been shown for the Serotonin transporter (5-HTT) with 5HTTLPR short allele carriers showing higher depressive symptoms in the presence of stressful life events but lower depressive symptoms in their absence (Caspi et al, 2003, Belsky et al, 2009). Significant associations were limited to the highly regulated and functionally active region surrounding NOS1's exon 2. The significant SNPs are in LD; therefore, it is difficult to suggest a mechanism by which these variants influence NOSI's ability to modulate stress responsiveness; however, rs3782218 bears further investigation due to its potential regulatory role.

\section{FUNDING AND DISCLOSURE}

Dr Elliott received consultancy fees from Cambridge Cognition and P1vital. Professor Deakin variously performed consultancy, speaking engagements, and research for Bristol-Myers Squibb, AstraZeneca, Eli Lilly, Schering Plough, Janssen-Cilag, and Servier (all fees are paid to the University of Manchester to reimburse them for the time taken); he has share options in P1vital. Professor Anderson has received consultancy fees from Servier and Alkermes, an honorarium for speaking from Lundbeck and grant support from Servier and AstraZeneca. The sponsors funded the work but had no further role in the design or conduct of the study or in the preparation, review, or approval of the manuscript. The remaining authors declare no conflict of interest.

\section{ACKNOWLEDGEMENTS}

The study was supported by the Sixth Framework Program of the European Union, NewMood, LSHM-CT-2004-503474, by the National Institute for Health Research Manchester Biomedical Research Centre, by the Hungarian Academy of Sciences (MTA-SE Neuropsychopharmacology and Neurochemistry Research Group), and by the National Development Agency (KTIA_NAP_13-1-2013-0001), Hungarian Brain Research Program-Grant No. KTIA_13_NAP-A-II/ 14. We are grateful to Diana Chase and Zoltan G. Tóth for their assistance in the recruitment and data acquisition, to Hazel Platt for her assistance in genotyping, and to Heaton Mersey Medical Practice and Cheadle Medical Practice for their assistance in the recruitment.

\section{REFERENCES}

Aquilano K, Baldelli S, Rotilio G, Ciriolo MR (2008). Role of nitric oxide synthases in Parkinson's disease: a review on the antioxidant and anti-inflammatory activity of polyphenols. Neurochem Res 33: 2416-2426.

Beach SR, Brody GH, Lei MK, Kim S, Cui J, Philibert RA (2014). Is serotonin transporter genotype associated with epigenetic susceptibility or vulnerability? Examination of the impact of socioeconomic status risk on African American youth. Dev Psychopathol 26: 289-304 1-16.

Belsky J, Jonassaint C, Pluess M, Stanton M, Brummett B, Williams R (2009). Vulnerability genes or plasticity genes? Mol Psychiatry 14: 746-754.

Benjamini Y, Hochberg Y (1995). Controlling the false discovery rate: a practical and powerful approach to multiple testing. $J R$ Stat Soc Series B 57: 289-300.

Berg DT, Gupta A, Richardson MA, O'Brien LA, Calnek D, Grinnell BW (2007). Negative regulation of inducible nitric-oxide synthase expression mediated through transforming growth factor-beta-dependent modulation of transcription factor TCF11. J Biol Chem 282: 36837-36844.

Bick J, Naumova O, Hunter S, Barbot B, Lee M, Luthar SS et al (2012). Childhood adversity and DNA methylation of genes involved in the hypothalamus-pituitary-adrenal axis and immune system: whole-genome and candidate-gene associations. Dev Psychopathol 24: 1417-1425. 
Bros M, Boissel JP, Godtel-Armbrust U, Forstermann U (2006). Transcription of human neuronal nitric oxide synthase mRNAs derived from different first exons is partly controlled by exon 1-specific promoter sequences. Genomics 87: 463-473.

Bros M, Boissel JP, Godtel-Armbrust U, Forstermann U (2007). The untranslated region of exon 2 of the human neuronal nitric oxide synthase (NOS1) gene exerts regulatory activity. Gene 405: $36-46$.

Brugha T, Bebbington P, Tennant C, Hurry J (1985). The list of threatening experiences: a subset of 12 life event categories with considerable long-term contextual threat. Psychol Med 15: 189-194.

Brugha TS, Cragg D (1990). The List of Threatening Experiences: the reliability and validity of a brief life events questionnaire. Acta Psychiatr Scand 82: 77-81.

Buchmann AF, Hellweg R, Rietschel M, Treutlein J, Witt SH, Zimmermann US et al (2013). BDNF Val 66 Met and 5-HTTLPR genotype moderate the impact of early psychosocial adversity on plasma brain-derived neurotrophic factor and depressive symptoms: a prospective study. Eur Neuropsychopharmacol 23: 902-909.

Butterworth P, Rodgers B, Windsor TD (2009). Financial hardship, socio-economic position and depression: results from the PATH Through Life Survey. Soc Sci Med 69: 229-237.

Caspi A, Sugden K, Moffitt TE, Taylor A, Craig IW, Harrington H et al (2003). Influence of life stress on depression: moderation by a polymorphism in the 5-HTT gene. Science 301: 386-389.

Das-Munshi J, Goldberg D, Bebbington PE, Bhugra DK, Brugha TS, Dewey ME et al (2008). Public health significance of mixed anxiety and depression: beyond current classification. $\mathrm{Br} J$ Psychiatry 192: 171-177.

Derogatis L (ed.) (1993). BSI: Brief Symptom Inventory: Administration, Scoring, and Procedures Manual. National Computer Systems Pearson Inc: Minneapolis.

Doucet MV, Harkin A, Dev KK (2012). The PSD-95/ nNOS complex: new drugs for depression? Pharmacol 133: $218-229$.

Fox J (2005). The R commander: a basic statistics graphical user interface to R. J Stat Software 14: 1-42.

Freeman B, Smith N, Curtis C, Huckett L, Mill J, Craig IW (2003). DNA from buccal swabs recruited by mail: evaluation of storage effects on long-term stability and suitability for multiplex polymerase chain reaction genotyping. Behav Genet 33: $67-72$.

Galecki P, Maes M, Florkowski A, Lewinski A, Galecka E, Bienkiewicz $M$ et al (2011). Association between inducible and neuronal nitric oxide synthase polymorphisms and recurrent depressive disorder. J Affect Disord 129: 175-182.

Gao SF, Qi XR, Zhao J, Balesar R, Bao AM, Swaab DF (2013). Decreased NOS1 expression in the anterior cingulate cortex in depression. Cereb Cortex 23: 2956-2964.

Hancock DB, Martin ER, Vance JM, Scott WK (2008). Nitric oxide synthase genes and their interactions with environmental factors in Parkinson's disease. Neurogenetics 9: 249-262.

Heim C, Binder EB (2012). Current research trends in early life stress and depression: review of human studies on sensitive periods, gene-environment interactions, and epigenetics. Exp Neurol 233: 102-111.

Hosang GM, Korszun A, Jones L, Jones I, McGuffin P, Farmer AE (2012). Life-event specificity: bipolar disorder compared with unipolar depression. Br J Psychiatry 201: 458-465.

Jaffrey SR, Snyder SH (1996). PIN: an associated protein inhibitor of neuronal nitric oxide synthase. Science 274: 774-777.

Joca SR, Guimaraes FS, Del-Bel E (2007). Inhibition of nitric oxide synthase increases synaptophysin mRNA expression in the hippocampal formation of rats. Neurosci Lett 421: 72-76.

Johnsen O, Skammelsrud N, Luna L, Nishizawa M, Prydz H, Kolsto $A B$ (1996). Small Maf proteins interact with the human transcription factor TCF11/Nrf1/LCR-F1. Nucleic Acids Res 24: 4289-4297.

Juhasz G, Chase D, Pegg E, Downey D, Toth ZG, Stones $\mathrm{K}$ et al (2009). CNR1 gene is associated with high neuroticism and low agreeableness and interacts with recent negative life events to predict current depressive symptoms. Neuropsychopharmacology 34: 2019-2027.

Lu L, Lin K, Qian Z, Li H, Cai Y, Li Y (2010). Predicting DNA methylation status using word composition. J Biomed Sci Eng 3: 672-676.

Luciano M, Huffman JE, Arias-Vasquez A, Vinkhuyzen AA, Middeldorp CM, Giegling I et al (2012). Genome-wide association uncovers shared genetic effects among personality traits and mood states. Am J Med Genet B Neuropsychiatr Genet 159B: 684-695.

Maciejewski PK, Prigerson HG, Mazure CM (2001). Sex differences in event-related risk for major depression. Psychol Med 31: 593-604.

Mathews DC, Henter ID, Zarate CA (2012). Targeting the glutamatergic system to treat major depressive disorder: rationale and progress to date. Drugs 72: 1313-1333.

McGuffin P, Knight J, Breen G, Brewster S, Boyd PR, Craddock N et al (2005). Whole genome linkage scan of recurrent depressive disorder from the depression network study. Hum Mol Genet 14: 3337-3345.

McManus S, Meltzer H, Brugha T, Bebbington P, Jenkins R (2009). Adult Psychiatric Morbidity in England, 2007: Results of a Household Survey. In: Statistics SC (ed.). The NHS Information Centre for Health and Social Care: London.

Meltzer $\mathrm{H}$, Bebbington P, Brugha T, Jenkins R, McManus S, Stansfeld S (2010). Job insecurity, socio-economic circumstances and depression. Psychol Med 40: 1401-1407.

Oliveira RM, Guimarães FS, Deakin JF (2008). Expression of neuronal nitric oxide synthase in the hippocampal formation in affective disorders. Braz J Med Biol Res 41: 333-341.

Pogun S, Kuhar MJ (1994). Regulation of neurotransmitter reuptake by nitric oxide. Ann N Y Acad Sci 738: 305-315.

Reif A, Kiive E, Kurrikoff T, Paaver M, Herterich S, Konstabel $\mathrm{K}$ et al (2011). A functional NOS1 promoter polymorphism interacts with adverse environment on functional and dysfunctional impulsivity. Psychopharmacology 214: 239-248.

Rijsdijk FV, Sham PC, Sterne A, Purcell S, McGuffin P, Farmer A et al (2001). Life events and depression in a community sample of siblings. Psychol Med 31: 401-410.

Rose EJ, Greene C, Kelly S, Morris DW, Robertson IH, Fahey C et al (2012). The NOS1 variant rs6490121 is associated with variation in prefrontal function and grey matter density in healthy individuals. Neuroimage 60: 614-622.

Russo MT, Donizetti A, Locascio A, D’Aniello S, Amoroso A, Aniello $\mathrm{F}$ et al (2004). Regulatory elements controlling Ci-msxb tissue-specific expression during Ciona intestinalis embryonic development. Dev Biol 267: 517-528.

Silva M, Aguiar DC, Diniz CR, Guimarães FS, Joca SR (2012). Neuronal NOS inhibitor and conventional antidepressant drugs attenuate stress-induced fos expression in overlapping brain regions. Cell Mol Neurobiol. 32: 443-453.

Steinert JR, Chernova T, Forsythe ID (2010). Nitric oxide signaling in brain function, dysfunction, and dementia. Neuroscientist 16: $435-452$.

Ursini G, Bollati V, Fazio L, Porcelli A, Iacovelli L, Catalani A et al (2011). Stress-related methylation of the catechol-O-methyltransferase Val 158 allele predicts human prefrontal cognition and activity. J Neurosci 4: 31.

Wang Y, Goligorsky MS, Lin M, Wilcox JN, Marsden PA (1997). A novel, testis-specific mRNA transcript encoding an NH2terminal truncated nitric-oxide synthase. J Biol Chem 272: 11392-11401. 
NOSI polymorphisms affect depression risk

JE Sarginson et al

Weber H, Klamer D, Freudenberg F, Kittel-Schneider S, Rivero O, Scholz CJ et al (2013). The genetic contribution of the NO system at the glutamatergic post-synapse to schizophrenia: further evidence and meta-analysis. Eur Neuropsychopharmacol 24: 65-85.
Zhou QG, Zhu LJ, Chen C, Wu HY, Luo CX, Chang L et al (2011). Hippocampal neuronal nitric oxide synthase mediates the stress-related depressive behaviors of glucocorticoids by downregulating glucocorticoid receptor. J Neurosci 31: 7579-7590.

Supplementary Information accompanies the paper on the Neuropsychopharmacology website (http://www.nature.com/npp) 